and right to left ventricular diameter ratio (Terarecon, California, USA) to diagnose pulmonary hypertension. Pulmonary infarct volume and perfused blood volume relative enhancement were also calculated (Syngo via, Siemens Healthineers, Forchheim, Germany). All radiologic parameters were correlated with clinical data. To assess if in situ thrombosis could be visualised on CT, isolated segmental and subsegmental filling defects were used as an imaging surrogate. For statistical analyses, Graphpad Prism9 and IBM SPSS v27.0 software were used.

Results The incidence of either central PE or DVT was equal between patients with COVID-19 and influenza pneumonia (20\%). The incidence of isolated segmental and subsegmental filling defects was higher in COVID-19 but without statistical significance $(44 \%$ vs $32 \% ; \mathrm{p}=0.5607)$. Right to left ventricular diameter and pulmonary artery to aorta ratios were higher in COVID-19 compared to influenza (1.01 vs 0.866 and 1.04 vs $0.904 ; \mathrm{p}=0.0071$ and $\mathrm{p}=0.0023$, respectively).

Conclusion In a comparable group of patients with severe COVID-19 and influenza pneumonia, CT features of pulmonary hypertension are more often present in patients with COVID-19 pneumonia despite an equal clot burden on CT. This is not attributable to pulmonary thrombosis visible on CT and supports the hypothesis that micro- rather than macrovascular obstruction is the cause of severe hypoxia in COVID-19 pneumonia.

\section{P95 ELEVATED D-DIMERS IN COVID-19 PATIENTS PREDICT PE BUT CAUTION IS NEEDED WITH HIGHER THRESHOLDS}

IJ Walker, 'R Hughes, ${ }^{2}$ A Ainley. 'Respiratory Medicine, Queen's Hospital, Barking, Havering and Redbridge University Hospitals NHS Trust, London, UK; ${ }^{2}$ Respiratory Medicine, King George Hospital, Barking, Havering and Redbridge University Hospitals NHS Trust, London, UK

\subsection{6/thorax-2021-BTSabstracts.205}

Introduction and Objectives COVID-19 is associated with a pro-inflammatory, hypercoagulable state, increasing the likelihood of developing pulmonary embolism (PE). Higher Ddimer levels have been noted in COVID-19 patients compared to the general population, which may lead to over-investigation by computed tomography pulmonary angiography (CTPA) if traditional thresholds (positive $\geq 0.5 \mathrm{mg} / \mathrm{L}$ ) are used. We aimed to investigate whether a higher D-dimer threshold could be used.

Methods A retrospective observational study was performed at Barking Havering and Redbridge University Hospitals NHS Trust from April 2020 - March 2021. The study included a cohort of confirmed/suspected cases of COVID-19 requiring hospital admission. D-dimer level on admission, CTPA outcome and requirement for intensive care unit (ICU) admission were analysed to assess D-dimer as a predictor of $\mathrm{PE}$ and clinical outcome in COVID-19.

Results In 404 patients included, mean D-dimer was $3.03 \mathrm{mg} /$ L. $186(46 \%)$ underwent CTPA, 32 (17\%) of which detected PE. In those with PE, mean D-dimer was $(8.62 \mathrm{mg} / \mathrm{L})$, significantly higher than those without $\mathrm{PE}(2.55 \mathrm{mg} / \mathrm{L}) \quad(\mathrm{P}=$ $<0.0001)$. Patients admitted to ICU had a significantly higher D-dimer $(4.35 \mathrm{mg} / \mathrm{L})$ than those who were not $(2.69 \mathrm{mg} / \mathrm{L})(\mathrm{P}$ $=0.049$ ). Applying the traditional threshold of $0.5 \mathrm{mg} / \mathrm{L}$ resulted in a sensitivity of $97 \%$ and specificity of $10 \%$ for detecting PE. Using higher thresholds of $1.0 \mathrm{mg} / \mathrm{L}$ and $2.0 \mathrm{ml} / \mathrm{L}$ resulted in sensitivity of $87 \%$ and $71 \%$, and specificity of $37 \%$ and $69 \%$, respectively.

Conclusions Our data strongly suggests that higher D-dimer levels are associated with disease severity e.g. complication with PE and requirement for ICU admission. Caution is needed as higher thresholds of $2.0 \mathrm{ml} / \mathrm{L}$ or greater, as suggested in previous studies, ${ }^{1}$ would have resulted in an unacceptably low sensitivity in this cohort. Our study highlights the need for further work evaluating use of adjusted D-dimer thresholds in patients with acute COVID-19 to aid decision making and help balance the risks of radiation associated with CTPA and consequences associated with missed diagnosis of PE.

\section{REFERENCE}

1. Ventura-Díaz $S$, et al. A higher D-dimer threshold for predicting pulmonary embolism in patients with COVID-19: a retrospective study. Emerg Radiol. 2020;27 (6):679-689. doi:10.1007/s10140-020-01859-1

\section{P96 PULMONARY VASCULAR DISEASE IN COVID-19: INSIGHTS FROM ARTIFICIAL INTELLIGENCE ANALYSIS IN A LARGE MULTICENTRE IMAGING DATABASE}

J Rossdale, P Charters, R Foley, W Brown, T Burnett, R Mackenzie Ross, J Suntharalingam, J Rodrigues. Royal United Hospitals Bath NHS Foundation Trust, Bath, UK

\subsection{6/thorax-2021-BTSabstracts.206}

Aims and Objectives An increased incidence of pulmonary thrombosis (PT) and right ventricular (RV) dysfunction is reported in COVID-19. The clinical significance is not fully understood and there are few large, multicentre studies. The National Covid-19 Chest Imaging Database (NCCID) was analysed for prevalence of PT in COVID-19 patients; we hypothesised associations between macroscopic PT, severity of parenchymal disease, evidence of RV dysfunction on CT and mortality.

Methods NCCID is a multicentre UK-wide centralised database comprised of radiological images from hospitalised COVID-19 patients. 391 thoracic contrast CT scans from 14 centres across England and Wales performed between 2nd March 2020 - 10th September 2020 underwent automated post-processing software (IMBIO LLC.) to determine RV:LV diameter ratio. Scans were manually reported for PT and quantitatively scored for arterial obstruction and severity of parenchymal involvement using CT- Severity Scoring (CT-SS)[1]. Imaging metrics were analysed for association with PT and 30 day mortality.

Results Automated RV:LV analysis was successful in 90\% (351/ 391) of scans. Mean age: 64, 53\% (186/351) male. Mortality data was available for 325 patients: 22 died within 30 days of scan $(6.7 \%(22 / 325))$.

Macroscopic PT was present in 16\% (56/351). Median Qanadli score was 6\% (IQR 3\%-17.5\%), indicating low burden arterial obstruction. PT was not associated with mortality $(\mathrm{p}=0.18)$.

RV:LV >1 on CT was observed in 59\% (206/351) (mean $R V: L V$ 1.08). RV:LV was significantly higher in the presence of PT (mean RVLV 1.17 vs $1.06 \mathrm{p}=0.011, \chi^{2}(2)=6.499$ ). $\mathrm{RV}: \mathrm{LV}$ was not predictive of mortality (AUC 0.467 , CI 0.358 $0.576)$.

CT-SS significantly predicted mortality (AUC 0.787, $\mathrm{p}=<0.0005$, CI 0.693-0.881). However there was no correlation between severity of parenchymal involvement and RV:LV 